\title{
Electrical Kindling Rat Model Preparation and Analysis of EEG Signals
}

\author{
Li-juan SHI ${ }^{1}$, Yi YU ${ }^{1,{ }^{*}}$, Yun $\mathrm{ZHAO}^{1}$, Liu-yang $\mathrm{XU}^{1}$, Xin-qi HE ${ }^{1}$ and \\ Jun-tang LIN \\ ${ }^{1}$ Xin Xiang Medical University, Henan,China \\ *Corresponding author,Email:shilijuancumt@163.com
}

Keywords: Epilepsy, EEG signals, Electric stimulation, Bispectrum analysis.

\begin{abstract}
Epilepsy (Epilepsy, EP) is a brain functional disorder caused by brain neurons abnormal discharge, however, the etiology and pathogenesis of epilepsy is still unclear. With the development of computer technology and digital signal processing technology, research and analysis of EEG signals is gradually applied to epilepsy. At present, a lot of epileptic rat models are adopted by the model for drugs, but they has a high fatality rate Here we Take SD rats as experimental objects, creatively using electrical stimulation method of preparation rat model of epilepsy, brain neurons in the rat hippocampus of discharge signal acquisition by Cerebus ${ }^{\mathrm{TM}} 64$ channel data acquisition system, and carries on the bispectrum analysis and processing, in order to find the effective characterization of epileptic rat brain electrical signal characteristics. The results of the study show that when rats had epileptic discharge, significantly enhanced the EEG signal is non Gaussian and non-linear, double spectrum peak increased significantly, peaks higher energy, peak position, will tend to be more in the high frequency region; and compared with the detected spikes, field potential signals of double spectrum peaks in larger quantities, the energy spectrum peaks is also higher. Therefore, bispectrum characteristics of field potentials can be used as an effective standard to identify epilepsy.
\end{abstract}

\section{Introduction}

Epilepsy (Eplilepsy, EP) is a chronic recurrent brain dysfunction syndrome, is a brain functional disorder caused by brain neurons abnormal discharge[1], and the lesions were hippocampus temporal lobe. It occurs mainly in young people, for the first time in the young people under the age of 15 patients, the attack rates was as high as $62 \%$ [2], so the research of pathogenic mechanism of epilepsy is very important. In preparation for epilepsy model, most of current experiment used methods of lithium chloride - pilocarpine model, in analysis of eeg signals, we often use mainly wavelet transformation, principal component analysis, etc[3], in diagnosis and treatment of epilepsy eeg has played a huge role, we can use different methods to analyze eeg signals, including linear and nonlinear methods, because individual differences in epileptic eeg signals is significant, and different one has obvious nonstationary randomness, which makes the accurate extraction of eeg features become difficult in the biomedical signal processing. Linear analysis methods are often used in the biomedical signal processing techniques, it is also a much more mature method, mainly includes the time domain analysis and frequency domain analysis method, etc[4]. In this paper, we used high order statistics analysis based on bispectrum analysis, which is effect remarkable in processing with non-gaussian and nonlinear stochastic signal[5]. 


\section{Model Preparation and Evaluation}

\section{Model Preparation}

In this paper, we adopt the method of electrical stimulation to the preparate epilepsy rat model, realizing fastly light of epileptic rats, the principle of the fast ignition is repeated stimulation under the same intensity of current (or voltage) for one part of rats, which can make the strength increases gradually, and the rats can be further induced systemic activity of epilepsy. The mainly parts of electrical stimulation of the epileptic rats include the hippocampus, amygdala, globus pallidus and piriform cortex[6], while the hippocampus and the amygdala are most commonly used. In this paper, the main electrical stimulation part to induce epilepsy is hippocampus, the standard conditions of electrical stimulation are $1 \mathrm{~ms}$ current wavelength, $60 \mathrm{hz}$ frequency, duration of $1 \mathrm{~s}$, stimulate once a day, we usually think electrical stimulation below $10 \mathrm{hz}$ frequency is not easy to fire model, and frequency between 60 and $100 \mathrm{hz}$ can be see as the best stimulus intervals within 1 to $60 \mathrm{~s}$, the wavelength should be controlled within $10 \mathrm{~ms}$ [7], and studies have shown that the square wave stimulation can significantly reduce the stimulation of brain tissue damage.

The specific process of model preparation is as follows: the SD male rats should be fixed on the stereotaxic instrument, and cut the head skin along the center line of the head of rats, and then separate periosteum, We use cranial drill grinding in the midline position on the right side of the cortex of the skull ( $2.5 \mathrm{~mm}$ after anterior fontanelle, $2.5 \mathrm{~mm}$ near by the center line).After removing the part of the skull, remove to the dura mater with bend needle. Then fixed electrode on the stereotaxic instrument, and implant subdural $2.9 \mathrm{~mm}$ [8], glue and package with dental cement, so as to collect data for many times. The process of electrode implantation as shown as Figure 1:

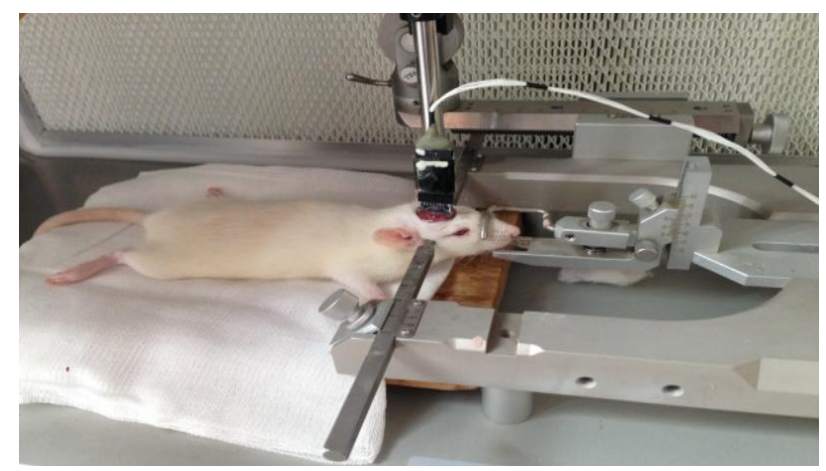

Figure. 1. The process of electrode implantation

\section{Model Evaluation}

The successfully established experimental rats will show the different levels of activity decreases, tics, tremor, scratching, nodding, "washing face", one side of the forelimbs clonus, and balance disorders[9].

\section{Signal Collection}

This article use CerebusTM 64 channel data acquisition system, realizing online real-time observation of eeg signals. The acquisition of eeg signals mainly includes spike potential and field potential LFP, spike potential uses $250 \mathrm{HZ}-5 \mathrm{KHZ}$ band pass filter, sample frequency is $30 \mathrm{ks} / \mathrm{s}$, while field potential LFP adopts $250 \mathrm{hz}$ low pass filter, and sample frequency is $1 \mathrm{ks} / \mathrm{s}$. 


\section{Data Processing}

Power spectrum estimation in frequency domain is transforming eeg signals from time domain to frequency domain[10], it will change eeg signal over time into that of over frequency, and then get the spectrum diagram to determine the frequency of the signal component based on the distribution of signal power spectrum in frequency domain. The effect is remarkable of the power spectrum analysis of the second order information, however, this algorithm for eeg analysis lost significantly higher order information, while the bispectrum analysis for processing with non-gaussian and nonlinear stochastic signal is remarkable based on higher order statistics analysis. Bispectrum analysis algorithm is one of the most simple spectral analysis technology of higher order, and double spectrum image also can be used as the obvious distinguish between different signal characteristics.

We mainly adopts double spectrum analys is method to analyze eeg signals based on the non-gaussian and nonlinear characteristics of eeg signal[11], and before analysis, we need to pre-process eeg data, in order to reduce the computational complexity of the signal, reduced the frequency of sampling field potential to $100 \mathrm{hz}$; and the spike potential signal frequency is reduced to $300 \mathrm{hz}[12]$, Then use filter filter and the bispectrum analysis. Bispectrum analysis of the parameter estimation method mainly includes direct method and indirect method, the function of the direct method is bispecd, while the function of the indirect method biseci[13]. This article mainly uses the direct estimation method, the form of function and parameter is: [bspec, waxis] = bispecd $(y, n f f$, wind, sampseg, overlap $)$; [14].

\section{Data Analysis}

The picture of the eeg signal analyzed by MATLAB is shown as Figure 2, Figure 3, Figure 4, and Figure 5:

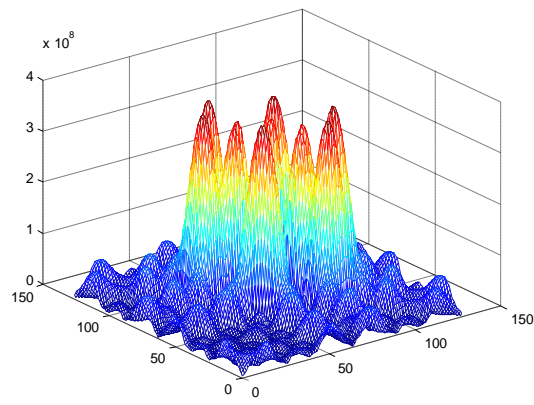

Figure. 2. Epilepsy Spike

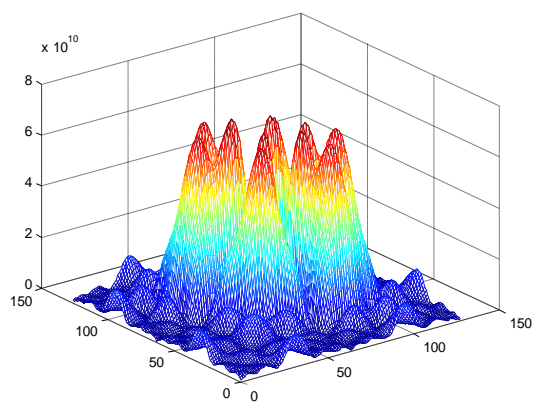

Figure. 3. Epilepsy Cortex 


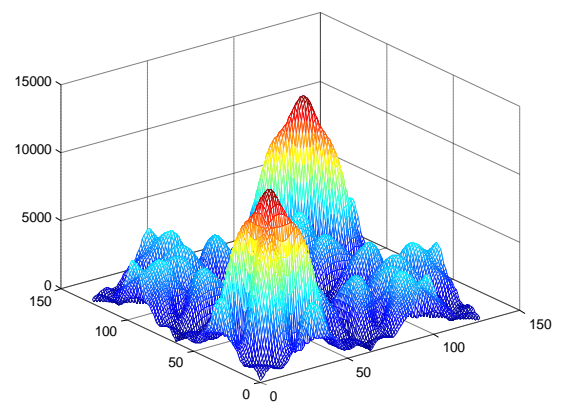

Figure. 4. Normal Spike

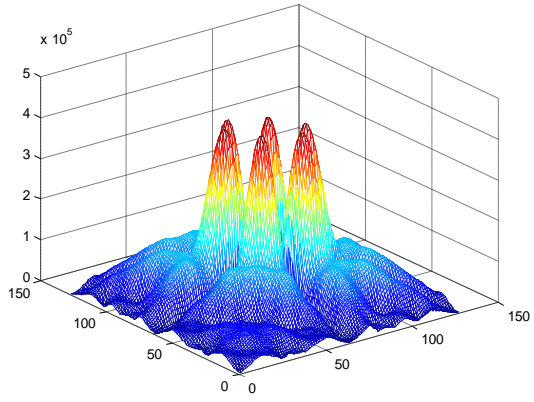

Figure. 5. Normal Cortex

For cortex and hippocampus of epilepsy rats, the results of bispectrum analysis are significantly different[15], when the rats had epileptiform discharge, the non-gaussian and nonlinear of eeg signal enhanced obviously, and peak increased obviously, the position of spectral peak also tend to high frequency area[16], and comparing with the spike potential, and also spectral peak of bispectrum increased, at the same time energy is also higher. Therefore, the bispectrum characteristics can be used as identification of epileptic. It shows that the bispectrum analysis of free rats and epilepsy rats can be used as the difference between normal eeg signals and that of epilepsy.

\section{Conclusions}

This paper, the preparation of epileptic rat model is by means of electrical stimulation, and electrodes are implanted in the head of the prepared rat model, after pretreatment, after preprocessing, we can found that the eeg signal is quite different in normal rats and epileptic rats by bispectrum analysis. Therefore, double spectrum characteristics can be used as an important basis in the actual clinical use to judge epilepsy, This is the meaning and purpose of this study.

\section{Acknowledge ment}

The experiment has spent three months, during this time, so many people give me help as much as possible, when I face some challenges, it's them give me the strength. At the beginning, when prepare some rats, they are so active that it is difficult to record the eeg signal, while we help each other as a team , and we work together to find a best way to analysis the data, what's more our school provide us with an experiment platform, and our teacher give us a lot of guidance, what is exciting, a good result has been developed. The success of this experiment will motivate us to do more research in the future!

\section{References}

[1]You M. Influence of expression flunarizine to the Pgp and Glu in brain tissues of amygdaloid kindling rat model[J]. Guizhou Medical Journal,2009.

[2]Zhang Y, Ji X, Zhang Y. Classification of EEG signals based on AR model and approximate entropy[C], 2015.

[3]Acharjee P P, Shahnaz C. Multiclass epileptic seizure classification using time-frequency analys is of EEG signals[C] ,2012:260-263. 
[4]Ai L, Huang L, Huang Y, et al. Study of Epileptic Electroencephalogram Using Bispectrum Analysis[J]. Journal of Xian Jiaotong University, 2004, 23:9-12,4.

[5]Liu Y, Zhou Y, Xin W, et al. Bispectrum analysis for feature extraction of pitting fault in wind turbine gearbox[C], 2010:1298-1301.

[6]Li S, Yi L. Feature Extraction of Lung Sounds Based on Bispectrum Analysis[C], 2010:393-397.

[7]Regan D, Mukherjee P, Seery D. General CMB bispectrum analysis using wavelets and separable modes[J]. Physical Review D Particles \& Fields, 2013, 88(4):395-418.

[8]Wu H, Li X, Guan X. Networking property during epileptic seizure with multi-channel EEG recordings[C]// International Conference on Advances in Neural Networks. Springer-Verlag, 2006:573-578.

[9]Wu H, Xia Y, Lai Y, et al. Study of Epileptic Rat's EEG Using Bispectrum Analysis[C]// First International Conference on Neural Interface and Control, 2005. Proceedings. 2005:119-122.

[10] Mohite N, Shastri R, Deosarkar S, et al. Epileptic electroencephalogram classification $[\mathrm{C}] / /$ International Conference on Communications and Signal Processing. 2014:467-471.

[11]Chua K C. Analysis of cardiac and epileptic signals using higher order spectra[J]. Queensland University of Technology, 2010.

[12]Xu L. The effects of Methoxamine on behavior and cortical EEG of epileptic rats induced by penicillin[J]. Stroke \& Nervous Diseases, 2011.

[13]Han L, Tang D Q. The study on anticonvulsant and antiepileptic effect of $\alpha$-asarone[J]. Journal of Jinggangshan Medical College, 2009. 